\title{
Identificación de saberes ancestrales en la etnia Emberá Katío sobre el cuidado del medioambiente
}

\author{
Elizabeth Cantero Galarcio ${ }^{1{ }^{10}}$, Elías Hernández Hernández ${ }^{(0)}$ \\ Universidad de Córdoba, Colombia
}

Autor de correspondencia: ${ }^{1}$ ecanterogalarcio@correo.unicordoba.edu.co Recibido: 04 de agosto de 2020 Revisado: 29 octubre de 2020 Aprobado: 05 de enero de 2021 Publicado: 18 de mayo de 2021

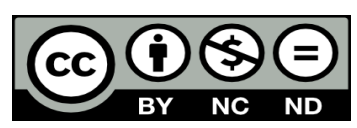

\section{Resumen}

La educación en Colombia tiene dificultades para reconocer el valor de los saberes ancestrales de las comunidades indígenas. Por lo tanto, le es difícil equilibrar los programas educativos con los requerimientos culturales de cada comunidad y, en consecuencia, estos saberes son excluidos de los programas educativos. El objetivo de este trabajo es identificar los saberes ancestrales de la cultura Emberá Katío de Tierralta, Córdoba, relacionados con el cuidado del medioambiente, para proponer una estrategia etnoeducativa en el área de ciencias naturales dirigida al grado quinto de básica primaria. La metodología del estudio se enmarca en el enfoque cualitativo, con un diseño etnográfico. La recolección de información se realizó con las técnicas de observación no participante, entrevista semiestructurada y grupo focal. La muestra estuvo conformada por dos docentes emberá y cinco indígenas, actores claves dentro de la comunidad —el jaibaná, el alguacil, el yerbatero, el cazador y el anciano-. Del análisis de resultados emergen cinco categorías - territorio, plantas medicinales, alimentación, chagra y memorias - con las que se diseña una estrategia etnoeducativa para la educación intercultural en el área de ciencias naturales y educación ambiental en el grado quinto de básica primaria, pero que puede ser replicada en otros cursos.

Palabras clave: población indígena, conocimientos tradicionales, educación intercultural, ciencias naturales, educación ambiental 


\title{
Identification of ancestral knowledge in the Emberá Katío ethnic group on environmental care
}

\begin{abstract}
Education in Colombia faces difficulties in the recognition of ancestral knowledge of indigenous communities. Therefore, it is difficult to balance educational programs and the cultural requirements of each community, resulting in the exclusion of this knowledge from educational programs. The objective of this article is to identify the ancestral knowledge of the Emberá Katío culture of Tierralta, Córdoba, related to the environmental care, in order to propose an ethno-educational strategy in the area of natural sciences for the fifth grade of primary school. The methodology of the study is based on a qualitative approach, with an ethnographic design. Data collection was carried out using non-participant observation, semi-structured interview, and focus group techniques. The sample consisted of two Emberá teachers and five indigenous people, key actors within the community: the shaman, the guardian, the herbalist, the hunter, and the elder. Five categories emerge from the analysis of the results-territory, medicinal plants, food, vegetable patch, and memories-with which an ethno-educational strategy is designed for intercultural education in the area of natural sciences and environmental education in the fifth grade of primary school, which can be replicated in other years.
\end{abstract}

Keywords: indigenous people, traditional knowledge, intercultural education, natural sciences, environmental education

\section{Identificação de conhecimentos ancestrais na etnia Emberá Katío sobre cuidados ambientais}

\section{Resumo}

A educação na Colômbia tem dificuldades em reconhecer o valor do conhecimento ancestral das comunidades indígenas. Portanto, é difícil equilibrar os programas educacionais e as exigências culturais de cada comunidade sendo este conhecimento excluído dos programas educacionais. O objetivo deste trabalho é identificar os conhecimentos ancestrais da cultura Emberá Katío de Tierralta, Córdoba, relacionados ao cuidado do meio ambiente, a fim de propor uma estratégia etno-educativa na área das ciências naturais voltada para a quinta série do ensino fundamental. A metodologia do estudo é inserida na abordagem qualitativa, com um desenho etnográfico. A coleta de dados foi realizada com as técnicas de observação não-participante, entrevista semi-estruturada e grupo focal. A amostra consistiu em dois professores emberá e cinco indígenas, atores-chave dentro da comunidade - o xamã, o guarda, o herborista, o caçador e o ancião. Da análise dos resultados, emergem cinco categorias - território, plantas medicinais, alimentos, chácara e memórias - com as que se desenha uma estratégia etno-educativa para a educação intercultural na área das ciências naturais e educação ambiental na quinta série do ensino fundamental, mas que pode ser replicada em outras séries.

Palavras-chave: povos indígenas, conhecimentos tradicionais, educação intercultural, ciências naturais, educação ambiental 
La trasformación cultural de los pueblos indígenas en Colombia ha sido una constante desde la colonización. Es producto de la coexistencia forzosa de varios grupos humanos con visiones políticas, sociales, culturales y religiosas diferentes (Friede, 1979). Las imposiciones culturales externas han ocasionado una lucha de las comunidades para preservar sus conocimientos ancestrales y la tierra, y para combatir la pobreza, a través de la exigencia de la reivindicación de sus derechos (Panqueba, 2012). Su objetivo principal es mantener la identidad cultural a través de diversas actividades, luchas territoriales, manifestaciones y reclamos políticos desde la década de los 70 (Jimeno, 1996). En la historia reciente, en los procesos de resignificación cultural de los pueblos indígenas, ha cobrado importancia el quehacer ambiental (Quintero \& Cardona, 2016) y la educación, como herramientas para evitar la destrucción de sus territorios ancestrales y el incumplimiento de los acuerdos pactados con los entes gubernamentales.

En este sentido, algunas de las luchas ganadas por parte de las comunidades indígenas implican el derecho a la educación propia y el reconocimiento a la pluriculturalidad. El problema radica en que el reconocimiento y aceptación de la pluriculturalidad de la población indígena en sus respectivos territorios han sido procesos muy lentos, que afectan especialmente el escenario educativo, incluso con la variedad de reformas jurídicas y constitucionales legales, ya que existen vacíos en los reglamentos de la cultura mayoritaria y las de los grupos étnicos cuando no hay una demarcación clara de la diferencia (Arbeláez \& Vélez, 2008). Por ello, "la educación intercultural en sí solo tendrá significación, impacto y valor cuando esté asumida de manera crítica, como acto pedagógico-político que procura intervenir en la refundación de la sociedad" (Walsh, 2010, p. 4).

Es así como la investigación es pertinente en cuanto a la evidente exclusión didáctica y pedagógica que enfrentan los niños y niñas indígenas del país, en especial los de la etnia Emberá Katío de la sede Volador de la Institución Educativa Los Morales, Tierralta, departamento de Córdoba. La problemática fue evidenciada a través de la observación previa de las características de las prácticas docentes en clase de ciencias naturales y educación ambiental [CN y EA] en el grado quinto. Se pudo observar que las prácticas educativas actuales no desarrollaban estrategias etnoeducativas que visibilicen los saberes ancestrales indígenas. Desde las directivas, falta gestión para la transformación del currículo hacia una escuela intercultural, donde todos los docentes en estos contextos estén direccionados a prácticas educativas dialogantes entre los saberes ancestrales indígenas y los de las comunidades no indígenas, para establecer puntos de encuentro que posibiliten el desarrollo de un eje temático. Esto es perjudicial, pues interfiere en la adaptación de los niños emberá al sistema educativo, dificulta la adquisición de conocimientos e incrementa la deserción escolar.

Las características principales de esta problemática son la desintegración de las prácticas culturales y tradicionales de los niños y niñas indígenas, como también la contribución a la pérdida de los conocimientos tradicionales. A esto se suma el racismo y la exclusión por parte de algunos de sus compañeros, lo que trae como consecuencia la desmotivación, el mal rendimiento académico y la deserción de los niños y niñas indígenas (Cabanzo, 2020).

Otras regiones de Colombia no son ajenas a esta problemática, a pesar de que en 1991, en el marco de una nueva constitución, se instituyó por primera vez en la historia, que 
Colombia es un país intercultural. A partir de entonces, se protegería la multiculturalidad y se respetarían las costumbres y tradiciones de las comunidades indígenas y las demás minorías. De ahí en adelante, surgieron otras normas que defienden la educación intercultural. Así, empezó a tomar fuerza el término etnoeducación, que Romero (2006) define como el proceso a través del cual los miembros de un pueblo internalizan, construyen conocimientos, desarrollan habilidades y destrezas de acuerdo con sus necesidades, que les permitan desempeñarse adecuadamente en su medio y proyectarse con identidad propia hacia otros grupos humanos. Sin embargo, la realidad es que la educación en Colombia tiene dificultades para satisfacer las necesidades de las poblaciones indígenas y equilibrar los programas educativos con los requerimientos culturales de cada comunidad.

Al poco reconocimiento de la cultura, de los conocimientos, de las experiencias y de los saberes ancestrales o tradicionales en el contexto educativo, se une la sede Volador, que tiene 974 estudiantes, desde el grado primero hasta once, de los cuales el $60 \%$ está en estado de vulnerabilidad. Los alumnos de primaria tienen entre 5 y 13 años; los de bachillerato, de 10 a 18 años. En la institución hay 30 alumnos de la etnia Emberá Katío, víctimas de desplazamiento forzado, con dificultades para adaptarse a la dinámica escolar. Cinco de estos niños han desertado en los últimos meses. En distintas reuniones institucionales y autoevaluaciones, se resalta la necesidad de incorporar al currículo ejes temáticos y metodologías etnoeducativas, como también la necesidad de capacitar a los docentes que ejercen su función en la educación intercultural.

En este punto, hay que hacer un bosquejo de lo que es la etnia Emberá Katío. La comunidad se ubica en el departamento de Córdoba, sobre los ríos Sinú, Esmeralda y Verde, en la región noroccidental del departamento de Antioquia y en los departamentos de Chocó, Caldas y Putumayo, con diversos asentamientos en Panamá y Ecuador. Se les conoce, desde antes de la colonización, como los indígenas de Chocó, personas que comparten espacio, cosmovisión, organización política y económica (Castillo, 2015). En Córdoba, el resguardo se ubica a $150 \mathrm{~km}$ del municipio de Tierralta, río arriba desde Puerto Frasquillo. Está conformado por cerca de 7000 habitantes, incluyendo las familias que viven en el casco urbano de Tierralta y zonas veredales (Vallejo \& Majore, 2018).

En cuanto a la estructura social y educativa, la familia está conformada por padre, madre e hijos: es la unidad básica productora. Viven en tambos - vivienda propia-construidos por los miembros de la familia, y las actividades cotidianas se extienden a otros miembros de la familia o comunidad cuando la labor es pesada. En el resguardo Emberá del alto Sinú, la comunidad lleva a cabo un proceso de educación propia hasta quinto de primaria. La educación infantil contempla tres ejes fundamentales: matemáticas, territorio y cosmovisión, impartidas por educadores de la comunidad Emberá que se preparan fuera del resguardo para impartir clases dentro de la comunidad (Castillo, 2015).

Los Emberá Katío son un pueblo espiritual. De acuerdo con su pensamiento colectivo, todo lo que habita sobre la tierra tiene "espíritu". En su área ancestral existen los lugares prohibidos, encantados y comunales. Los lugares prohibidos son aquellos donde no se pueden llevar a cabo actividades de pesca, caza, o corte de madera. En estos lugares habita el creador. Sitios como los cementerios, ríos, montañas, lagos, lagunas, quebradas, sitios de origen - lugar donde la comunidad se estableció desde tiempos inmemoriales- y 
manglares se consideran prohibidos. Los sitios comunales son aquellos donde se realizan las actividades cotidianas, como los tambos, áreas de pesca o caza (Vallejo \& Majore, 2018).

Además, la trasmisión del conocimiento para los Emberá es la tradición oral. Los niños aprenden la lengua materna hasta los cuatro años. Al llegar a la escuela, adoptan el español como segunda lengua. Sin embargo, no todos los miembros de la etnia han logrado acceder a la educación propia y es baja la tasa de alfabetización de la comunidad. En el resguardo del alto Sinú, la comunidad doza — de donde son la mayoría de los estudiantes de la sede Volador - habla la lengua nativa emberá bédea y una fracción menor habla castellano. Aunque no existen cifras concretas sobre la tasa de analfabetismo, es de interés general que los miembros de la comunidad accedan a programas de educación (Vallejo \& Majore, 2018).

También hay que mencionar el desplazamiento forzado. La incursión de grupos armados y la construcción de la represa Urra (Castañeda, 2012) han ocasionado que los niños y niñas deban integrarse a la comunidad escolar a edades inusuales para el grado a cursar. La falta de bases necesarias o de rutina escolar dificulta que los estudiantes adquieran nuevos conocimientos. La situación se agrava cuando la institución educativa en la que están inscritos no cuenta con las políticas, el personal y la logística necesarios para brindar la etnoeducación a la que tienen derecho, como es el caso de la sede Volador. Es así como los niños emberá adquieren conocimientos foráneos en todas las áreas de educación contempladas - en especial en ciencias naturales-y desconocen sus saberes ancestrales, lo que genera un sesgo en el aprendizaje de esta población, ya que los niños y niñas obtienen de forma deficiente tanto los conocimientos occidentales como los nativos por la falta de bases escolares y por la ausencia de integración de los saberes ancestrales en el sistema educativo al cual se pretenden integrar.

En este caso, los niños y niñas emberá en edad escolar se ven obligados a interiorizar una cultura totalmente ajena, sin las bases necesarias para afrontar la pérdida de sus usos, costumbres ancestrales y el desarraigo territorial. De esta forma, el proceso de escolarización fortalece los aspectos científicos requeridos por la población en general, pero desconoce los procesos culturales de la comunidad Emberá Katío.

Todos estos argumentos revelan una problemática en la que los niños y niñas de la etnia Emberá Katío en la vereda Volador se enfrentan a un proceso de occidentalización de su cultura, puesto que la institución educativa no implementa el enfoque diferencial requerido por esta población, ya que es evidente que el docente no desarrolla estrategias o metodologías acordes al contexto en el que se relacionan niños y niñas de varias culturas. No existe un programa que facilite la etnoeducación y, en el caso de la EA, se estaría perdiendo la conexión de los niños y niñas indígenas con su entorno propio de la comunidad Emberá, lo que generaría un desarraigo de los futuros adultos con la naturaleza y el desconocimiento de su modo de vida y el cuidado de la naturaleza.

Es imperioso resaltar que, para mejorar la calidad educativa de los niños y niñas de cualquier etnia, deberían poder beneficiarse de los procesos etnoeducativos a los cuales tienen derecho. No obstante, estos programas solo se implementan en algunos planteles pertenecientes a modelos de educación propia. Por ello, se plantea el siguiente interrogante de investigación: ¿¿de qué manera los saberes ancestrales de la etnia Emberá Katío sobre el cuidado del medioambiente contribuyen a generar una estrategia etnoeducativa para el área de $\mathrm{CN}$ y 
EA del grado quinto - básica primaria - en la sede Volador de la Institución Educativa Los Morales, Tierralta, Córdoba? Por lo tanto, el objetivo de esta investigación fue identificar los saberes ancestrales de la etnia Emberá Katío sobre el cuidado del medioambiente, con el fin de integrarlos a la generación de estrategias etnoeducativas para el área de ciencias naturales, enfocadas en niños de grado quinto de dicha institución.

\section{Metodología}

Esta investigación se sustenta en el paradigma interpretativo-hermenéutico, que de acuerdo con Espinosa et al. (2011), "parte de reconocer la diferencia existente entre los fenómenos sociales y naturales, buscando la mayor complejidad y el carácter inacabado de los primeros, que están siempre condicionados por la participación del hombre" (p. 106). En este sentido, sus intereses primordiales apuntan al significado del quehacer humano y de la vida social. Es una interpretación reflexiva, en este caso de los saberes ancestrales de la etnia Emberá Katío sobre el cuidado del medioambiente, con el propósito de proponer una estrategia etnoeducativa para el área de $\mathrm{CN}$ y EA del grado quinto.

Dado el paradigma y naturaleza del estudio, la investigación se enmarca en el enfoque cualitativo, ya que es abierta y flexible. Se construye durante el trabajo de campo, dentro de las fases de estudio, y está orientada a describir y analizar los conocimientos tradicionales de la comunidad Emberá, así como los significados que la comunidad le da a sus saberes. Finalmente, se presenta los resultados de manera que se resalten las regularidades que implica un proceso cultural.

\section{Participantes}

La muestra consistió en dos docentes que conforman el equipo de educación en la comunidad doza de indígenas Emberá Katío y cinco integrantes representativos de la comunidad doza: el jaibaná, el alguacil, el hierbatero, un cazador y un miembro de los más ancianos.

\section{Categorías de análisis}

En esta investigación se han establecido categorías conceptuales y de análisis que emergen con base en los siguientes objetivos específicos:

- Caracterizar los saberes ancestrales de la etnia Emberá Katío del alto Sinú, en Tierralta, Córdoba, en la comunidad doza sobre el cuidado del medioambiente.

- Diseñar una estrategia etnoeducativa desde los saberes ancestrales de la etnia Emberá Katío sobre el cuidado del medioambiente para el área de CN y EA del grado quinto.

Estos objetivos permiten alcanzar el objetivo general y, por consiguiente, se puede responder la pregunta de investigación. De las categorías, con base en las observaciones realizadas, surgieron las dimensiones que fueron evaluadas por los indicadores planteados en la tabla 1, enfocadas en las prácticas ambientales. A continuación, se detallan las categorías, sus dimensiones e indicadores y la asociación que tienen con los objetivos de la investigación (tabla 1). 
Tabla 1

Categorías, dimensiones e indicadores

\begin{tabular}{|c|c|c|c|}
\hline $\begin{array}{l}\text { Objetivos } \\
\text { específicos }\end{array}$ & Categorías & Dimensiones & Indicadores \\
\hline \multirow{3}{*}{1} & \multirow{3}{*}{$\begin{array}{l}\text { En el caso de las tablas, } \\
\text { es preferible hacer un } \\
\text { salto de renglón en vez } \\
\text { de romper la palabra } \\
\text { (claro está, cuando el } \\
\text { espacio lo permite). }\end{array}$} & Conocimientos & $\begin{array}{l}\text { Tienen conocimiento del medioam- } \\
\text { biente y la importancia de su cui- } \\
\text { dado. }\end{array}$ \\
\hline & & Prácticas & $\begin{array}{l}\text { Las prácticas son en relación con } \\
\text { la utilización y conservación del } \\
\text { medioambiente. }\end{array}$ \\
\hline & & Rituales & $\begin{array}{l}\text { Practican rituales en favor a la pro- } \\
\text { tección y respeto de la naturaleza. }\end{array}$ \\
\hline 2 & $\begin{array}{l}\text { Estrategia etnoeduca- } \\
\text { tiva }\end{array}$ & Didáctica & $\begin{array}{l}\text { Estructuración de una propuesta que } \\
\text { integre los saberes ancestrales al } \\
\text { plan educativo de CN y EA. }\end{array}$ \\
\hline
\end{tabular}

\section{Técnicas de recolección de información}

Para el cumplimiento del primer objetivo específico, se hizo necesario recolectar la información por medio de la técnica de observación no participante. Se pretendió detallar lo observado - concerniente al medioambiente- en el viaje y durante tres semanas de estadía en la comunidad doza. Los datos obtenidos se registraron en un diario de campo. En seguida, se empleó la técnica de entrevista, cuyo objetivo fue la recolección de datos a partir de las propias palabras, pensamientos y reflexiones de los sujetos entrevistados —en este caso los dos docentes-, con el propósito de caracterizar los saberes ancestrales sobre el cuidado del medioambiente. Además, se empleó la técnica de grupo focal - la cual consistió en una discusión mediante preguntas semiestructuradas - con el jaibaná, el alguacil, el hierbatero, un cazador y uno de los miembros de la comunidad más ancianos, los cuales son guiados por un moderador - los investigadores-, quien conduce la sesión según una guía.

\section{Técnicas de análisis de información}

Para el análisis se empleó la técnica de rejilla de análisis (Feixas \& Cornejo, 1996), que se trata de un instrumento muy flexible que puede adaptarse a diversas áreas de aplicación: evaluación individual, grupal, familiar, entre otros. La rejilla es una tabla donde, de acuerdo con las categorías y dimensiones, se plasma un resumen de las respuestas y en la siguiente columna se hacen los análisis correspondientes según los puntos de encuentro, el contraste con la teoría o los antecedentes de la investigación.

\section{Resultados}

\section{Entrevista con docentes}

El hábitat o territorio, las memorias ancestrales, la cosmovisión de armonía y equilibrio entre la naturaleza y el hombre son principios transversales para la vida de la etnia Emberá Katío. Se llegó a la anterior reflexión al entrevistar a dos maestros de la comunidad doza. En las entrevistas se revelan importantes saberes como el del territorio. Según los docentes, la perspectiva que tiene el Emberá Katío sobre el medioambiente es análoga a su territorio, ya que es el lugar donde habitan. 
Por ello, es necesario que el tema territorio se incorpore al plan de área de $\mathrm{CN}$ para explicar a los niños y niñas distintos temas como ecosistemas, medioambiente, entre otros, para lograr una inclusión al currículo y fomentar estos saberes desde la etnoeducación, lo que debería ser beneficioso para los estudiantes indígenas al sentirse incluidos en el proceso de enseñanza-aprendizaje, y también para que los estudiantes no indígenas conozcan los saberes ancestrales de sus compañeros. Los docentes entrevistados concuerdan en la relación estrecha entre el territorio y la identidad emberá. Esto es apoyado por Macías y Lorieth (2020), cuando en su investigación concluyen que "tanto la construcción de territorialidad como lo comunitario poseen un concepto conector: el del vínculo. Así, tanto la apropiación identitaria y sociocultural de la relación con la naturaleza parte de los vínculos que se generan en el territorio" (p. 178).

Es evidente, entonces, la especial importancia que tiene el territorio para los emberá. Según los entrevistados, es su medioambiente donde adquieren lo necesario para su subsistencia como la alimentación, la pintura de sus maquillajes, los elementos para la construcción de sus artesanías, tambos y canoas. Es la fuente principal de la obtención de plantas medicinales. Desde el análisis de resultados de la entrevista a los docentes y al triangularlos con la observación, emergen categorías como territorio, huertos o chagra y alimentación, las cuales, mediante un proceso pedagógico dirigido al desarrollo de competencias en $\mathrm{CN}$ y EA, se pueden incluir en estrategias didácticas acordes al contexto intercultural donde se apliquen. Sánchez (2015) resalta la necesidad de promover el aprendizaje desde los saberes ancestrales en pro de mejorar el proceso de enseñanza-aprendizaje de los estudiantes indígenas. Estos saberes deben ser adaptados de forma sistemática y progresiva al currículo.

\section{Caracterización de los saberes ancestrales}

La interpretación de los saberes ancestrales y los conceptos presentados son producto de los testimonios extraídos del grupo focal. Estos actores claves, esenciales para su comunidad, además de poseer un amplio conocimiento sobre costumbres y tradiciones, han alcanzado diversos grados de escolaridad, por lo cual estaban en la capacidad de compartir versiones simplificadas de sus conocimientos como se observa a continuación.

El territorio doza es un escenario amplificador de saberes, de tradición, de experiencias, de mitos, de leyendas y, lo más importante, de reflexiones frente a los puntos de encuentro entre dichos saberes, que emergen de sus principales actores - jaibaná, alguacil, hierbatero, cazador y anciano-, este grupo de personas, a través de sus conocimientos, puede propiciar una propuesta etnoeducativa de integración al plan de área de CN y EA, desde donde se recreen ambientes óptimos de aprendizaje en contextos interculturales. Para este propósito, se identificaron puntos de encuentro entre las respuestas del grupo focal según las dimensiones - conocimiento, prácticas y rituales- de la categoría saberes ancestrales sobre el cuidado del medioambiente. Para presentar reflexiones y aportar nuevos datos, se reconocen cinco categorías emergentes: territorio, plantas medicinales, alimentación, chagra y memorias, las que, desde la observación y la entrevista a docentes, empiezan a tener relevancia en relación con las CN y EA.

En la reunión, los representantes emberá expusieron una extraordinaria descripción del medioambiente - o como lo llaman ellos: territorio- que es el medio natural sagrado donde viven, así como de los terrenos que fueron modificados por el proyecto de la hidroeléctrica 
URRA y por los colonos dedicados a los cultivos ilícitos. De igual forma, resaltan elementos ancestrales como plantas medicinales, alimentos, ritos, costumbres, formas de trabajar en las chagras, cacería y pesca. Detalles más nobles como artesanías, maquillajes, mitos, leyendas, construcción de tambos, cercados, canoas, quedaron relatados con extraordinarios detalles. Todo este análisis y las reflexiones permiten definir cada una de las categorías emergentes de la siguiente forma:

Territorio. Es el lugar donde habitan los Emberá Katío, que ancestralmente es considerado como sagrado, ya que les fue otorgado por su dios Caragabi, pero con límites establecidos. Es caracterizado por una gran movilidad sobre extensas áreas en las que se realiza la agricultura, la cacería y la pesca. El territorio está compuesto por varios ecosistemas - terrestres y acuáticos-. Los ríos son los más representativos de los ecosistemas acuáticos. Para ellos, la riqueza se traduce en "pescado y territorio". Esta información es relevante ya que la analogía medioambiente/ territorio que perciben los indígenas puede ser utilizada en el aula de clases de ciencias naturales para explicar a los niños y niñas distintos temas como ecosistemas, medioambiente, entre otros, para lograr una inclusión al currículo y practicar la etnoeducación.

Plantas medicinales. Se evidencia su utilización ancestral en la comunidad Emberá Katío, ya que por medio de ellas se puede, en muchos casos, salvar la vida de alguien que habite en territorios lejanos. Además, permiten la superación de enfermedades y dolencias con recursos locales y saberes propios. Sin embargo, a causa de la occidentalización, esta práctica se está perdiendo. Por ello, es necesario rescatar estos saberes desde la escuela, con el propósito de proponer proyectos de cultivo e información de cada planta y sus propiedades. Así se muestran las plantas medicinales como un componente más del manejo completo de la salud y el territorio.

Alimentación. Por las características de la etnia Emberá Katío, su alimentación se basa en los productos que están disponibles en su territorio. Como cultivos, se encuentran el plátano, el arroz y el maíz. Esta información permite proponer el rescate de alimentos ancestrales y resaltar la importancia de cada especie animal y el desequilibrio que sucede en un ecosistema si llegase a desaparecer. Esto tiene el propósito de cultivar conciencia ambiental en los indígenas. No quiere decir que se les esté quitando o se está interfiriendo en sus prácticas ancestrales de caza; más bien, es incentivar en ellos producción intelectual en la cual propongan estrategias de uso racional y sostenible.

Chagra. Estos pequeños cultivos o huertas se encuentran cerca del tambo. Están al cuidado principalmente de mujeres, para la producción de plantas medicinales y alimentos que constituyen, en la actualidad, importantes aportes para el desarrollo sostenible de las comunidades que aprovechan los recursos naturales, en una relación de cuidado del medioambiente. Esta categoría es relevante, ya que desde la escuela intercultural se pueden incentivar buenas prácticas agrícolas, ambientales y alimenticias con un proyecto de huerta escolar. En este ejercicio se pueden abordar temas importantes como la fotosíntesis, partes de la planta, la germinación, prácticas amigables con el medioambiente, entre otras.

Memorias. Cuando los Emberá hablan de memorias, se refieren a sus características ancestrales en torno a la cultura, rituales, la alimentación, bailes, cantos, vestidos, lengua, artesanías y algunas prácticas en relación con el cuidado de su territorio. Esta categoría permite un mayor desarrollo en la parte de las ciencias sociales, pero no se quiso dejar de 
lado en las ciencias naturales, por cuanto toca temas como los mitos o relatos de cómo se creó el mundo emberá, en especial la formación de los ríos. De ahí surge la importancia que le dan a esta fuente hídrica, por su diversidad de explotación.

De esta forma, quedan establecidas las categorías para trabajar en una propuesta que incorpore al plan de área de CN y EA los saberes ancestrales de los Emberá Katío sobre el medioambiente, con el propósito de promover transformaciones en las realidades de los niños y niñas de esta comunidad de la escuela Volador, pero no solo en los estudiantes mencionados, también en los estudiantes no indígenas u occidentales, ya que aprenden desde otras culturas combinadas con la de ellos, es decir, un diálogo de saberes pero con propósitos en común.

También hay que resaltar las transformaciones que debe tener el docente y las directivas frente a la etnoeducación en contextos interculturales, todo esto sustentado teóricamente por Arbeláez y Vélez (2008), Guzmán (2008), Meneses (2016), Palacios (2017), Patiño (2004), Rodríguez (2011) y Sosa (2014), entre otros, quienes apuntan a que la etnoeducación "es uno de los caminos para el afianzamiento y afrontar los ejercicios colectivos que desafíen estereotipos estructurales, que desde las representaciones sociales generan violencias que se recrean o se transforman para mantener diferentes formas de exclusión" (Palacios, 2017, p. 3). Esto demuestra que la etnoeducación se enfoca en la edificación conceptual del reconocimiento del ser, es decir, en el reconocimiento de la presencia de otras maneras de sentir, percibir y vivir en el tiempo. Por ello, según Palacios (2017), "desde la literatura con enfoque diferencial étnico y la oralitura ancestral, se presentan al estudiante caminos para que llegue a la comprensión de ser habitado por la multiculturalidad de un territorio y de los territorios" (p. 5).

\section{Diseño de la estrategia etnoeducativa}

La estrategia es el resultado del análisis de la caracterización de saberes ancestrales de la etnia Emberá Katío sobre el cuidado del medioambiente. El mencionado proceso surge de reflexiones profundas sobre la cosmovisión emberá previamente categorizada. En síntesis, estas categorías brindan una ruta temática del componente biológico y ambiental para abordar en el contexto educativo intercultural con la etnoeducación y se consolidará en cinco talleres. Para la integración al plan de área, la ruta que se propone sigue la secuencia: evidencia de aprendizaje, temas, motivación, preconceptos, explicación, video, producción intelectual, plenaria, indagación, socialización, reflexiones y diálogo con las ciencias naturales.

En la estrategia etnoeducativa diseñada se propondrán tres objetivos:

1. Promover la etnoeducación de forma práctica y de reflexión crítica sobre los temas de CN y EA.

2. Incrementar el quehacer pedagógico y didáctico de los maestros mediante la inclusión de nuevas prácticas pedagógicas que reconozcan los saberes ancestrales sobre el medioambiente.

3. Promover la participación activa de los estudiantes en los procesos de enseñanzaaprendizaje a partir del reconocimiento de los saberes ancestrales sobre el medioambiente de la etnia Emberá Katío.

Los temas de los talleres serán: territorio, plantas medicinales, alimentación tradicional, chagra y memorias, con los cuales se buscará rescatar los saberes tradicionales e integrarlos 
al plan curricular, a través de herramientas didácticas, dibujos, historias y relatos.

Sin embargo, se necesitará emplear un método conocido que brinde la mejor ruta para desarrollar la estrategia etnoeducativa. En este sentido autores como Barbosa y Viviana (2016), Cáceres et al. (2014), Fresneda (2019), Muñoz (2018), Peña (2016), Riveros et al. (2014) y la Secretaría de Educación Distrital [SED] de Bogotá (2014) confirman que en este tipo de propuestas etnoeducativas y multiculturales es pertinente el método basado en la reflexión, la acción y la participación [RAP], que "concibe el conocimiento como una construcción colectiva y otorga un importante papel a los saberes populares" (SED, 2014, p. 31). Desde el escenario educativo, este método pone esos saberes, experiencias y habilidades en función de una transformación social, que involucra la acción -social, ambiental, cultural, religiosa, económica, entre otras- de los individuos y las comunidades.

De la misma forma, referentes como García (2017), Sánchez (2015), Olivares (2014), Gómez y Gómez (2006), Macías y Lorieth (2020), Uribe-Pérez (2019), Arias (2018) y Riveros et al. (2014) aportan a las propuestas. Ellos indican la importancia de educar en ambientes interculturales desde el diálogo de saberes indígenas y no indígenas. Al respecto Gómez y Gómez (2006) mencionan la correspondencia entre el sistema de conocimientos del método científico y el sistema de conocimientos de los saberes ancestrales tradicionales, a partir de la premisa de un diálogo intercultural, con la pretensión de propiciar la mutua fertilización de los dos sistemas de conocimientos.

En este sentido, la mediación del docente es esencial, como lo manifiesta Uribe-Pérez (2019), quien resalta que las estrategias empleadas por los profesores en las clases de ciencias deben considerar la pluralidad de cosmovisiones y la descolonización del saber en la práctica pedagógica. El otro escenario es la comprensión de la amplia experiencia natural emberá, que a su vez implica la interacción con sus realidades. Riveros et al. (2014) expone: "formas de cultivo de hierbas, plantas aromáticas y hortalizas en espacios pequeños como patios, balcones, terrazas o, incluso, en recipientes como macetas y cajones. Esta práctica de producción sigue vigente en las comunidades afrocolombianas en zonas rurales y urbanas" (p. 20). Al reflexionar frente a esto, se concluye que es una práctica valiosa para la educación en CN y EA, ya que se pueden rescatar estas prácticas ancestrales y convertirlas en estrategias educativas articuladas, pues se fomentan prácticas de desarrollo sostenible y cuidado ambiental (Quilaqueo et al., 2020).

La segunda práctica se relaciona con la alimentación. Por las características de la etnia Emberá Katío, su comida se basa en los productos que están disponibles en su territorio. Como cultivos se encuentran el plátano, el arroz y el maíz. El pescado sigue siendo el aporte proteico que más consumen, seguido del aprovechamiento del cerdo y de las gallinas. En este sentido, al recorrer la comunidad, se evidencian prácticas alimentarias comunitarias, a orilla del río, en el tambo y en el salón mayor. Por estas razones, es también una práctica resaltada, por cuanto se comunica con las $\mathrm{CN}$ para desarrollar estrategias educativas sobre la nutrición y todo lo que de esta se desprenda - sistema digestivo, tipos de alimentos, entre otros-. Según Vargas (2010), los conocimientos ancestrales pueden ser relacionados con la cultura, la alimentación, el medioambiente, la biología, la botánica, entre otros, para ser descubiertos y ponerlos en práctica en aulas etnoeducativas.

\section{Conclusiones}


Luego de la disertación de los resultados, se puede concluir que se obtuvo información valiosa en cuanto al conocimiento y al cuidado medioambiental que se han dado de generación en generación, entre otras informaciones. La integración de los saberes ancestrales en la asignatura CN será posible siempre y cuando los docentes cuenten con los conocimientos referentes a la comunidad de interés, en este caso la comunidad Emberá. El elemento más importante de la comunidad es el territorio. Por lo tanto, cualquier conocimiento que se pretenda impartir en los niños debe contemplar este eje fundamental para desglosar los demás temas de enseñanza. Las herramientas pedagógicas permitirán la actualización de los métodos de educación empleados con las comunidades indígenas. De esta forma, será posible brindarles educación de calidad sin ocasionar sismas entre sus conocimientos ancestrales y los nuevos conocimientos adquiridos.

\section{Referencias}

Arbeláez, J., \& Vélez, P. (2008). La etnoeducación en Colombia: una mirada indígena [Trabajo de grado, Universidad Eafit]. Repositorio Institucional Universidad Eafit. http://repository.eafit.edu.co/handle/10784/433

Arias, F. (2018). El diálogo de saberes entre las ciencias naturales y las cosmovisiones Indígenas acerca de la naturaleza [Tesis doctoral, Universidad Nacional de Colombia]. Repositorio Institucional UN. http://bdigital.unal.edu.co/70182/1/tesis_Frank_\%20Edisson_\%20 Arias.pdf

Barbosa, O., \& Viviana, Z. (2016). Desarrollo de la metodología (RAP) desde la interacción entre docentes y el apoyo de las Tecnologías de la Información y Comunicación (TIC), en el Colegio Carlos Albán Holguín IED [Trabajo de maestría, Universidad de los Andes]. Repositorio Uniandes. https://n9.cl/0k17

Cabanzo, M. (2020). El quehacer pedagógico, una reflexión para el ejercicio de la docencia con los Emberas Katíos. Revista Boletín Redipe, 9(8), 83-95.

Cáceres, D., González, C., Rivera, F., Mejía, A., Solano, R., Alpargatero, L., \& González, D. (2014). Orientaciones para el área de Educación para la Ciudadanía y la Convivencia. Alcaldía Mayor de Bogotá. https://n9.cl/vjww

Castañeda, A. (2012). ¿Son los Embera Katío del Alto Sinú étnicamente correctos? Pontificia Universidad Javeriana.

Castillo, L. (2015). Conflictos ambientales y movimientos sociales: el caso del movimiento embera katío en respuesta a la construcción de la represa Urrá (1994-2008). Memoria y sociedad, 19(39), 94-105. https://n9.cl/8jg0

Espinosa, C., Agudelo, L., \& Pachón, M. (2011). La hermenéutica en el desarrollo de la investigación educativa en el siglo XXI. Itinerario Educativo. Revista de la Facultad de Educación, 25(57), 101-120.

Feixas, G., \& Cornejo, J. (1996). Manual de la técnica de rejilla: mediante el programa RECORD V. 2.0. Paidós. http://diposit.ub.edu/dspace/handle/2445/33138

Fresneda, M. (2019). Evaluación y modelo pedagógico en el colegio Orlando Fals Borda. Universidad Externado de Colombia. https://bdigital.uexternado.edu.co/ 
handle/001/1594

Friede, J. (1979). Proceso de aculturación del indígena en Colombia. Revista Colombiana de Antropología, 22, 13-28.

García, D. (2017). Sistematización de experiencias: reconstrucción de la memoria histórica del Frente Nacional de Pueblos Indígenas (FRENAPI). Revista Latinoamericana de Derechos Humanos, 28(1), 117-146. https://n9.cl/3dy0v

Gómez, J., \& Gómez, G. (2006). Saberes tradicionales agrícolas indígenas y campesinos: rescate, sistematización e incorporación a la IEAS. Ra Ximhai, 2(1), 97-126. http://www. uaim.edu.mx/webraximhai/Ej-04articulosPDF/06\%202006.pdf

Guzmán, E. (2008). Etnoeducación y políticas educativas en Colombia: la fragmentación de los derechos. Revista Educación y Pedagogía, 20(52), 15-26.

Jimeno, M. (1996). Juan Gregorio Palechor: tierra, identidad y recreación étnica. Journal of Latin American Anthropology, 1(2), 46-77.

Macías, M., \& Lorieth, A. (2020). Sincronizando vínculos con la Tierra y la comunidad, a partir de diálogos de saberes ancestrales: sistematización de una experiencia en Bogotá [Trabajo de maestría, Universidad Pedagógica Nacional]. Repositorio Institucional UPN. http://repositorio.pedagogica.edu.co/handle/20.500.12209/11404

Meneses, Y. (2016). La etnoeducación afrocolombiana: conceptos, trabas, patriarcado y sexismo. A propósito de los 20 años de la Ley General de Educación 115 de 1994. Revista Historia de la Educación Latinoamericana, 18(27), 35-66.

Muñoz, Z. (2018). La ciudadanía ambiental una apuesta de construcción colectiva [Trabajo de maestría, Universidad de Ciencias Aplicadas y Ambientales] Repositorio UDCA. https://n9.cl/sazm4

Olivares, B. (2014). Sistematización del conocimiento ancestral y tradicional de la etnia kari ña en el estado Anzoátegui, Venezuela. Revista de Investigación, 38(82), 89-102. https:// dialnet.unirioja.es/descarga/articulo/4742845.pdf

Palacios, J. (2017). La etnoeducación en Antioquia, teoría y prácticas sin instrumentos. Implementación de políticas públicas etnoeducativas [Disertación doctoral, Universidad EAFIT]. Repositorio Institucional Universidad Eafit. https://n9.cl/c1 o6n

Panqueba, F. (2012). Elaboración y negociación de la pobreza indígena. En Clacso (Ed.), Las relaciones internacionales de la pobreza en América Latina y el Caribe (pp. 291-327). Clacso.

Patiño, P. (2004). Estado del arte de la etnoeducación en Colombia con énfasis en política pública. Ministerio de Educación Nacional.

Peña, E. (2016). La RAP, estrategia para la enseñanza de los derechos humanos y el territorio [Trabajo de maestría, Universidad Pedagógica]. Repositorio Institucional UPN. http:// repository.pedagogica.edu.co/handle/20.500.12209/3135

Quilaqueo, R., Sartorello, S., \& Torres, C. (2020).Diálogo de saberes en educación intercultural en educación intercultural: conflicto epistémico en contextos indígenas de Chile y México. Centro de Investigación en Educación en Contexto Indígena e Intercultural; Universidad 
Católica de Temuco.

Quintero, G., \& Cardona, A. (2016). Plan de gestión ambiental local a partir de la cosmovisión en la relación hombre-naturaleza de los indígenas Embera Katío de la comunidad de Sikuedo ubicada en el corregimiento Santa Cecilia Municipio de Pueblo Rico [Tesis Doctoral, Universidad Tecnológica de Pereira]. Red de repositorios latinoamericanos. https://repositorioslatinoamericanos.uchile.cl/handle/2250/142779.

Riveros, A., González, A., Grueso, A., \& Bustos, M. (2014). Etnociencia: perspectiva pedagógica de los estudios afrocolombianos para la enseñanza de las Ciencias Naturales. Alcaldía Mayor de Bogotá. https://n9.cl/qt37

Rodríguez, S. (2011). La política educativa (etnoeducación) para pueblos indígenas en Colombia a partir de la constitución de 1991 [Disertación doctoral, Universidad Nacional de Colombia]. Repositorio Institucional UN. https://n9.cl/kp0gx

Romero, F. (2006). Manuel Quintín Lame Chantre. El indígena ilustrado, el pensador indigenista. Revista Maguaré, 20, 257-264.

Sánchez, M. (2015). Los saberes ancestrales como aporte a la interculturalidad en los estudiantes de nivel básico superior del colegio particular Jorge Barek, cantón La Libertad, provincia de Santa Elena, año lectivo 2014-2015 [Trabajo de grado, Universidad Estatal Península de Santa Elena].Repositorio Dspace. https://repositorio.upse.edu.ec/xmlui/ handle/46000/2965

Secretaría de Educación Distrital de Bogotá. (2014). Currículo para la excelencia académica y la formación integral. Orientaciones para el área de Ciencias Naturales. Alcaldía de Bogotá. https://n9.cl/e9yt7

Sosa, T. (2014). Análisis de la política pública de etnoeducación y su relación con la construcción identitaria del Cabildo Muisca de Suba [Trabajo de grado, Universidad Piloto de Colombia]. Repositorio Institucional Universidad Piloto de Colombia. http:// repository.unipiloto.edu.co/handle/20.500.12277/8968

Uribe-Pérez, M. (2019). Saberes ancestrales y tradicionales vinculados a la práctica pedagógica desde un enfoque intercultural: un estudio realizado con profesores de ciencias en formación inicial. Revista Educación y Ciudad, 2(37), 57-71.

Vallejo, F., \& Majore, I. (2018). Los Emberá kdel Alto Sinú-Córdoba. Oraloteca, (9), 64-84. http://revistas.unimagdalena.edu.co/index.php/oraloteca/article/view/2901

Vargas, I. (2010). Conocimientos tradicionales: balances y perspectivas desde la propiedad intelectual. Universidad del Rosario.

Walsh, C. (2004). Colonialidad, conocimiento y diáspora afro-andina: construyendo etnoeducación e interculturalidad en la universidad. En E. Restrepo, \& A. Rojas (Eds.), Conflicto e (in)visibilidad Retos en los estudios de la gente negra en Colombia (pp. 331346). Editorial Universidad del Cauca. 